\title{
Correction to: Upper and Lower Values in Zero-Sum Stochastic Games with Asymmetric Information
}

\author{
Dhruva Kartik $^{1}$ (D) Ashutosh Nayyar ${ }^{1}$
}

Published online: 16 September 2020

(c) Springer Science+Business Media, LLC, part of Springer Nature 2020

\section{Correction to: Dynamic Games and Applications https://doi.org/10.1007/s13235-020-00364-x}

The original article has been published without funding information. This has been corrected with this erratum.

Acknowledgements This work was supported by NSF Grants ECCS 1509812 and ECCS 1750041 and ARO Award No. W911NF-17-1-0232.

Publisher's Note Springer Nature remains neutral with regard to jurisdictional claims in published maps and institutional affiliations.

The original article can be found online at https://doi.org/10.1007/s13235-020-00364-x.

$凶 \quad$ Dhruva Kartik

mokhasun@usc.edu

Ashutosh Nayyar

ashutosh.nayyar@usc.edu

1 Ming Hsieh Department of Electrical and Computer Engineering, University of Southern

California, Los Angeles, CA 90007, USA 